\title{
Vom Stillen zur Familienkost - (wie) ist Beikost ohne Brei möglich?
}

\author{
Prof. Dr. Mathilde Kersting, Forschungsinstitut für Kinderernährung Dortmund
}

\author{
Das Prinzip des Baby Led Weaning (BLW) geht davon aus, dass Säuglinge im 2. Lebens- \\ halbjahr selbst entscheiden, was und wie viel sie neben dem Stillen essen. Das Stillen \\ bildet weiterhin die Hauptnahrungsquelle, es wird durch Selbstbedienung des Säug- \\ lings aus der Familienkost ergänzt $[10,16]$.
}

Der früher im englischen Sprachgebrauch gängige Begriff weaning könnte leicht als Abstillen verstanden werden und dann mit den Absichten des BLW kollidieren. International ist weaning mehr und mehr durch den Begriff complementary feeding abgelöst worden, welcher eindeutiger die Ergänzung des Stillens bezeichnet, ähnlich dem deutschen Begriff Beikost.

\section{Prinzipien und Erwartungen}

Beim BLW soll sich der Säugling selbst mit Lebensmitteln füttern, die im Rahmen der Familienernährung bereitstehen. Mit einer solchen Handhabung der Beikost „nach Bedarf“ würden Vorteile des Stillens „nach Bedarf“ übernommen, bei dem sich die Milchbildung durch das Saugen des Kindes an der Brust physiologisch-hormonell gesteuert dem individuellen Bedarf des Kindes anpasst [2].

Muttermilch als alleiniges Nahrungsmittel in den ersten Lebensmonaten ist in ihrer Zusammensetzung auf den Bedarf des Säuglings zugeschnitten. Mit der Beikost kommt es dagegen darauf an, eine nach Art und Menge passende Auswahl verschiedener Lebensmittel zu treffen, die zusammen mit der verbleibenden Milch dem Nährstoffbedarf des Säuglings gerecht wird. Bei strikter Verfolgung des BLW-Prinzips müsste demnach eine ausgewogene Familienernährung, zum Beispiel nach dem Prinzip der Optimierten Mischkost [8], bereitgestellt werden und in ihrer Beschaffenheit, beispielsweise der Textur, den Essfertigkeiten des Säuglings entsprechen. Eine frühe Variation der angebotenen Lebensmittelpalette und die freie Auswahl durch den Säugling könnten die Akzeptanz einer ausgewogenen gemischten Kost längerfristig fördern [11].

\section{Die traditionelle Beikost - mit Brei}

\section{Lebensmittelbezogene Empfehlungen}

In Deutschland ist der „Ernährungsplan für das 1. Lebensjahr“ des Forschungsinstituts für Kinderernährung (FKE) seit Jahrzehnten als Standard in der Ernährungsberatung bewährt $[9,10]$ (Abb. 1). Er ist unter anderem Grundlage der Handlungsempfehlungen für die Ernährung von Säuglingen, die von der Initiative „Gesund ins Leben“ verbreitet werden

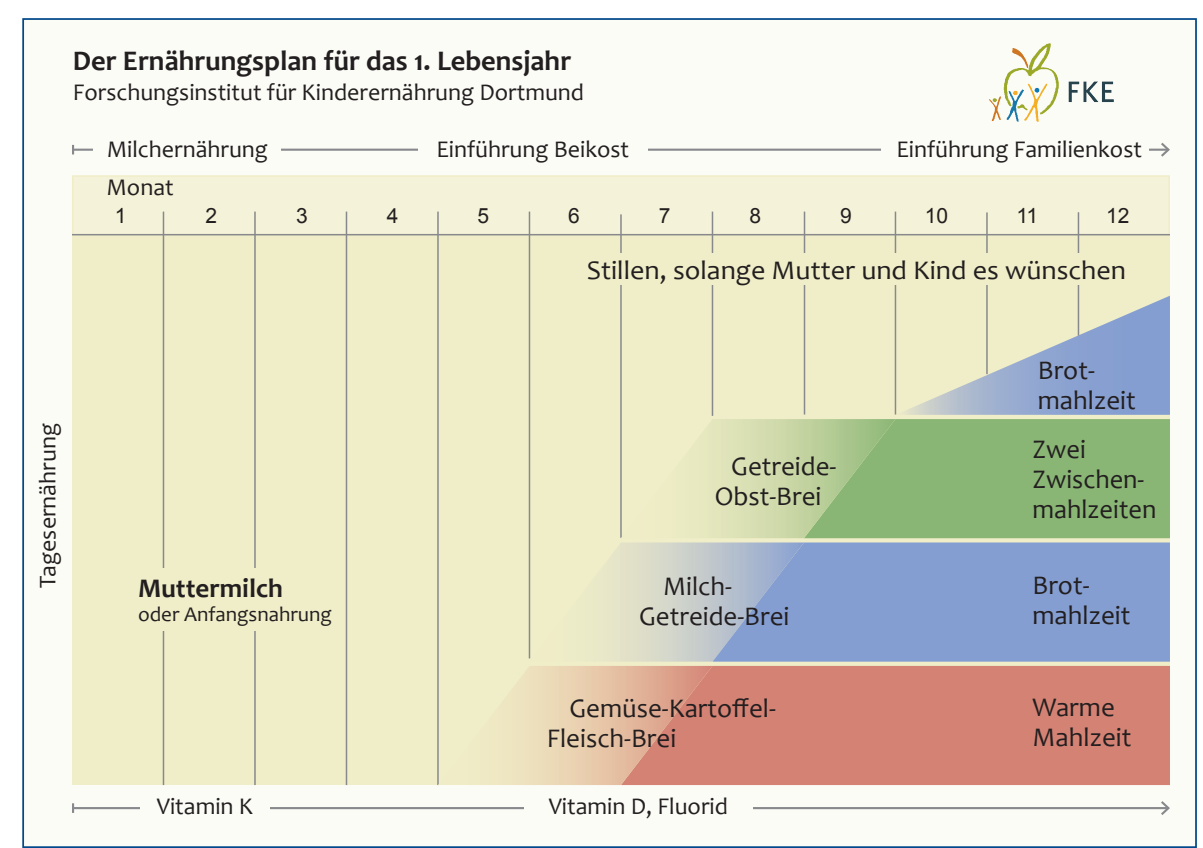

Abb. 1 @ Forschungsinstitut für Kinderernährung Dortmund 
unter Mitwirkung des multidisziplinären Netzwerks Junge Familie, in dem auch die Hebammen (DHV) vertreten sind.

Der „Ernährungsplan“ umfasst 3 Phasen, ausgerichtet am ernährungsphysiologischen Bedarf und der senso-motorischen Entwicklung der Kinder:

- ausschließliche Milchernährung in den ersten 4 bis 6 Lebensmonaten

- Einführung von Beikost-Breimahlzeiten ab dem 5. bis 7. Lebensmonat

- Einführung von Familienkost ab dem 10. Lebensmonat

Mit der Beikost werden in etwa monatlichen Abständen nacheinander drei aufeinander abgestimmte Breie eingeführt, die sich mit den verbleibenden Milchmahlzeiten wie in einem Baukastensystem zu einer ausgewogenen Tagesernährung ergänzen.

\section{Sicherung der Nährstoffzufuhr}

Die Nährstoffzufuhr mit dem „Ernährungsplan“ wurde berechnet und anhand der aktuellen Referenzwerte für die Nährstoffzufuhr der Deutschen Gesellschaft für Ernährung bewertet. Die EFSA hat dieses Vorgehen bei der Entwicklung lebensmittelbezogener Ernährungsrichtlinien als vorbildlich für die Kinderernährung in Europa herausgestellt [7]. So konnte gezeigt werden, dass bei Selbstherstellung der Beikost und fortgeführtem Stillen im 2. Lebenshalbjahr eine empfehlungsgerechte Nährstoffzufuhr erreicht wird [10]. Ausnahmen sind Jod und Eisen, zwei für die Entwicklung von Säuglingen und Kleinkindern bedeutende Nährstoffe.

Möglichkeiten zur Sicherung der Jodversorgung sind die Verwendung jodangereicherter kommerzieller Beikostbreie oder die Supplementierung [1]. Aufgrund des hohen Eisenbedarfs im 2. Lebenshalbjahr wird die Beikost mit einer fleischhaltigen Mahlzeit eingeführt, in den weiteren Mahlzeiten wird eisenreiches Vollkorngetreide mit Vitamin-Creichem Obst kombiniert, um die Eisen- bioverfügbarkeit zu verbessern. Dennoch finden sich bei einem Teil der Säuglinge, die gemäß „Ernährungsplan“ ernährt werden, im Alter von 10 Monaten Anzeichen für erschöpfte Eisenspeicher [6]. Eisen gilt europaweit als kritischer Nährstoff bei älteren Säuglingen und Kleinkindern [7]. Allerdings ist die Interpretation der üblichen Parameter des Eisenstatus im Verlauf des Säuglingsalters immer noch schwierig.

Der hohe Energiebedarf pro kg Körpergewicht im 2. Lebenshalbjahr erfordert eine hohe Energiedichte $(\mathrm{kcal} / \mathrm{g})$ der Beikost. Dieser Anforderung wird im „Ernährungsplan“ unter anderem durch den Milch-Getreide-Brei und durch einen Fettzusatz zu den anderen Beikostmahlzeiten Rechnung getragen.

\section{Die andere Beikost - mit Fingerfood}

\section{Studienergebnisse}

Das Baby Led Weaning wurde von einer britischen Hebamme und Stillberaterin entwickelt und in Büchern auch in deutscher Übersetzung bekannt gemacht [16]. In einem 2014 veröffentlichten strukturierten Literatur-Review des FKE wurden 11 Publikationen (von 7 Arbeitsgruppen) gefunden, die ganz unterschiedliche Fragen rund um das BLW untersuchten, zum Beispiel den Gewichtsstatus von Kindern mit BLW im Vergleich zu traditioneller Beikost, Einstellungen und Fütterverhalten von Eltern, Essfertigkeiten der Kinder. Auch eine systematische Übersichtsarbeit existierte, die sich mit der Machbarkeit des BLW befasste [2]. Kürzlich wurden zwei weitere Studien aus Neuseeland veröffentlicht, allerdings mit kleinen Kollektiven von 9 bis 26 Kindern in den einzelnen Füttergruppen $[3,15]$. In einer der beiden Studien wurden erstmals Berechnungen der Nährstoffzufuhr anhand von Ernährungsprotokollen vorgenommen [15]. Dabei nahm die BLWGruppe bei gleicher Energiezufuhr signifikant mehr Fett und weniger Mikronährstoffe (z.B. Eisen, Calcium, Vitamin
C) auf als die löffelgefütterte Gruppe. Typisch in dieser Studie ist, dass die Kinder in der BLW-Gruppe länger ausschließlich gestillt wurden und später Beikost erhielten. Bemerkenswert in dieser und anderen Studien ist weiterhin, dass die Mütter meist beide Füttermethoden, BLW und Löffelfütterung, nebeneinander angewendet hatten.

\section{Interpretation}

Anhand der derzeitigen Studienlage ist es nicht möglich, die tatsächliche Ernährung und Entwicklung der Kinder bei strikter Anwendung des BLW in der Praxis zu bewerten. Da es keine verbindliche Definition des BLW gibt, ordneten sich die Mütter in den überwiegend beobachtenden Studien selbst der BLW-Gruppe zu oder wurden nachträglich in die Studiengruppen eingeteilt.

Bei Befolgung des BLW-Prinzips ist der Beikostanteil an der Ernährung bis weit in das 2. Lebenshalbjahr hinein gering und Milch bleibt das Hauptnahrungsmittel. Theoretisch bestehen bei später Einführung der Beikost Risiken für die Nährstoffzufuhr, da beim verlängerten ausschließlichen Stillen im 2. Lebenshalbjahr die Zufuhr von Eisen und dann auch von weiteren Nährstoffen kritisch wird [5]. Muttermilch ist aufgrund des geringen Eisengehaltes keine relevante Eisenquelle, trotz guter Bioverfügbarkeit. Bei Beikost in Form von Fingerfood gibt es nur wenige Fleischspeisen, die Säuglinge problemlos kauen und schlucken können. Eisenangereichter oder jodangereichter Brei kommt bei Fingerfütterung nicht infrage. Eine Verzögerung der Beikosteinführung ist auch aus Sicht der Allergieprävention nicht empfehlenswert.

\section{Beikost als Brei und Fingerfood - Vorteile vereinigen}

Ausschlaggebend für die Handhabung der Beikost sollten der individuelle Ent- 
wicklungsstatus des Kindes und die Einbettung in ein ernährungsphysiologisch abgesichertes Ernährungskonzept sein.

\section{Senso-motorische Entwicklung}

Die Entwicklung der Essfertigkeiten bei Säuglingen und Kleinkindern verläuft interindividuell sehr variabel [12]. Deshalb ist im „Ernährungsplan“ seit jeher ein Zeitfenster im 5. bis 7. Monat für die Einführung der Beikost vorgesehen, das sich bei den nächsten Entwicklungsschritten fortsetzt. Die meisten Kinder können mit 5 bis 6 Monaten problemlos Brei essen, manche aber erst mit 9 Monaten. In einer ähnlichen Spannbreite entwickelt sich das Selberessen. Einige Kinder beginnen schon im Alter von 5 bis 6 Monaten damit, Lebensmittel wie Brot in den Mund zu nehmen, manche aber erst mit 1 Jahr. Die Entwicklung des Selberessens und damit der sozialen Teilhabe an der Familienernährung sollte auch bei breigefütterten Säuglingen gefördert werden. Dass die meisten Eltern dies intuitiv umsetzen, lässt sich aus den BLWStudien interpretieren, bei denen die Mütter häufig beide Füttermethoden nebeneinander eingesetzt hatten.

\section{Lebensmittelvariation}

Beim Baby Led Weaning wird postuliert, dass die Kinder später beim Essen weniger wählerisch seien. Dies konnte bisher zwar nicht durch Studien belegt werden. Bei herkömmlicher Breifütterung wurde aber gezeigt, dass täglicher Wechsel der Gemüsesorte bei Einführung der Beikost die Akzeptanz neuer Lebensmittel nicht nur in der Beikostphase, sondern bis in das Kindesalter anhaltend förderte [13].

Wenn sich Säuglinge neben der Löffelfütterung, die weiterhin die Ernährungsgrundlage bleibt, auch selbst mit geeigneten Lebensmitteln am Familientisch füttern, kann sich die haptische und sensorische Vielfalt bei der Ernährung erweitern. In herkömmlicher Beikost ist die Variation der Zutaten gering [14]. In 
kommerzieller Gläschenkost, aber auch bei Selbstherstellung des Gemüse-Kartoffel-Fleisch-Breis, werden hauptsächlich Karotten verwendet, mit Abstand gefolgt von Tomaten, Pastinaken und Kürbis.

\section{Fazit}

Das mit dem Baby Led Weaning angestrebte Idealziel, die Eigensteuerung der Nahrungsaufnahme beim Stillen mit der Beikost fortzuführen, ist als Konstrukt interessant, hat sich aber in strikter Form gegenüber dem natürlichen Verlauf der senso-motorischen Entwicklung der Kinder offensichtlich nicht durchsetzen können. Die Erkenntnisse aus den vorliegenden Studien zum BLW, wonach die Löffelfütterung und das Selberfüttern häufig nebeneinander praktiziert werden, können ein willkommener Anlass für eine Lockerung in der Handhabung der Beikost sein. Denn ausschließliche Breifütterung ist ebenso wenig kindgerecht wie ausschließliches Fingerfood. Die Kombination von beidem, je nach Entwicklungsstand des Kindes und mit entsprechendem Augenmaß der Eltern, wird dem Kind als Individuum bei der Ernährung am ehesten gerecht.

Das Baby Led Weaning kann Denkanstöße für eine Öffnung der Breiempfehlungen im „Ernährungsplan“ geben. Beikost als Fingerfood und die traditionelle Breieinführung schließen einander nicht aus. Auf diese Weise können die Vorteile der Ernährung nach dem „Ernährungsplan" (ausreichende Energie- und Nährstoffzufuhr) und des Baby Led Weaning (frühzeitige Gewöhnung an sensorisch vielfältige Lebensmittel) einander ergänzen zum Vorteil der gesundheitsförderlichen Kinderernährung.

\section{Literatur}

1 Alexy U, Drossard C, Kersting M, Remer $\mathrm{T}$. Iodine intake in the youngest: impact of commercial complementary food. Eur J Clin Nutr 2009; 63: 1368-1370

2 Cameron SL, Heath AL, Taylor RW. How feasible is Baby-led Weaning as an approach to infant feeding? A review of the evidence. Nutrients 2012; 4 : 1575-1609

3 Cameron SL, Tayler RW, Heath AL. Development and pilot testing of Baby-Led Introduction to SolidS - a version of Baby-Led Weaning modified to address concerns about iron deficiency, growth faltering and choking. BMC Pediatrics 2015; 15: 99 DOI 10.1186/ s12887-015-0422-8

4 Daniels L, Heath AL, Williams SM, Cameron SL, Fleming EA, Taylor BJ, Wheeler BJ, Gibson RS, Taylor RW. Baby-Led Introduction to SolidS (BLISS) study: a randomised controlled trial of a baby-led approach to complementary feeding. BMC Pediatr. 2015; 15: 179. doi: 10.1186/s12887-015-0491-8

5 Dewey KG. Nutrition, growth, and complementary feeding of the breastfed infant. Pediatr Clin North Am 2001; 48: 87-104

6 Dube K, Schwartz J, Mueller MJ, Kalhoff $\mathrm{H}$, Kersting M. Iron intake and iron status in breastfed infants during the first year of life. Clin Nutr 2010; 29 : 773-778

7 EFSA NDA Panel (EFSA Panel on Dietetic Products, Nutrition and Allergies). Scientific Opinion on nutrient requirements and dietary intakes of infants and young children in the European Union. EFSA Journal 2013; 11 (10): 3408, 103 pp

8 Forschungsinstitut für Kinderernährung (Hrsg) Empfehlungen für die Ernährung von Kindern und Jugendlichen - die Optimierte Mischkost. FKE, Dortmund, 2014, www.fke-do.de

9 Forschungsinstitut für Kinderernährung (Hrsg) Empfehlungen für die Ernährung von Säuglingen. FKE, Dortmund, 2015, www.fke-do.de

10 Hilbig A, Lentze M, Kersting M. Einführung und Zusammensetzung der Beikost. Monatsschrft Kinderheilkd 2012; 11: 1089-1095
11 Kersting M, Hilbig A, Disse S. Säuglingsernährung und Geschmacksprägung. Einfluss früher sensorischer Erfahrungen auf die kindliche Ernährung. Monatsschr Kinderheilkd 2015; 163: 783-789

12 Largo R. Wachstum und Entwicklung. In: Lentze M, Schaub J, Schulte F, Spranger F (Editors). Pädiatrie Grundlagen und Praxis, Springer Verlag: Berlin 2007: 8-62

13 Maier-Nöth A, Schaal B, Leathwood P, Issanchou $S$. The Lasting Influences of Early Food-Related Variety Experience: A Longitudinal Study of Vegetable Acceptance from 5 Months to 6 Years in Two Populations. PLoS ONE 2016; 11(3): e0151356. doi:10.1371/journal. pone.0151356

14 Mesch C, Stimming M, Foterek K, Hilbig A, Alexy U, Kersting M, Libuda L. Food variety in commercial and homemade complementary meals for infants in Germany - market survey and dietary practice. Appetite 2014; 76: 113-119

15 Morison BJ, Taylor RW, Haszard JJ, et al. How different are baby-led weaning and conventional complementary feeding? A cross-sectional study of infants aged 6-8 months. BMJ Open 2016; 6: e010665. doi:10.1136/bmjopen-2015-01066

16 Rapley G, Murkett T. Baby-led Weaning - das Grundlagenbuch. 2013, München: Kösel-Verlag

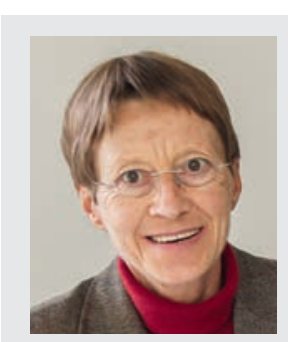

Prof. Dr. Mathilde Kersting Universitätskinderklinik Bochum Forschungsinstitut für Kinderernährung Heinstück 11 44225 Dortmund E-Mail: kersting@fke-do.de 